\title{
Paired immunoglobulin-like receptor B (PIR-B) inhibits BCR-induced activation of Syk and Btk by SHP-1
}

\author{
Akito Maeda ${ }^{1}$, Andrew M Scharenberg ${ }^{2}$, Satoshi Tsukada ${ }^{3}$, Joseph B Bolen ${ }^{4}$, Jean-Pierre Kinet ${ }^{2}$ \\ and Tomohiro Kurosaki*,1 \\ ${ }^{1}$ Department of Molecular Genetics, Institute for Liver Research, Kansai Medical University, Moriguchi 570-8506, Japan; \\ ${ }^{2}$ Laboratory of Allergy and Immunology, Beth Israel Hospital and Harvard Medical School, Boston, Massachusetts, 02215, USA, \\ ${ }^{3}$ Department of Medicine III, Osaka University Medical School, Osaka 565, Japan; ${ }^{4}$ Hoechst Marion Roussel, Bridgewater, New \\ Jersey, 08807, USA
}

Coligation of paired immunoglobulin-like receptor $B$ (PIR-B) with B cell antigen receptor (BCR) blocks antigen-induced $B$ cell activation. This inhibition is mediated in part by recruitment of SHP-1 and SHP-2 to the phosphorylated ITIMs in the cytoplasmic domain of PIR-B; however the molecular target(s) of these phosphatases remain elusive. Here we show that PIR-B ligation inhibits the BCR-induced tyrosine phosphorylation of $\operatorname{Ig} \alpha / \operatorname{Ig} \beta$, Syk, Btk and phospholipase C (PLC)$\gamma 2$. Overexpression of a catalytically inactive form of SHP-1 prevents the PIR-B-mediated inhibition of tyrosine phosphorylation of Syk, Btk, and PLC- $\gamma 2$. Dephosphorylation of Syk and Btk mediated by SHP-1 leads to a decrease of their kinase activity, which in turn inhibits tyrosine phosphorylation of PLC- $\gamma 2$. Furthermore, we define a requirement for $L y n$ in mediating tyrosine phosphorylation of PIR-B. Based on these results, we propose a model of PIR-B-mediated inhibitory signaling in which coligation of PIR-B and BCR results in phosphorylation of ITIMs by Lyn, subsequent recruitment of SHP-1, and a resulting inhibition of the BCR-induced inositol 1,4,5-trisphosphate generation by dephosphorylation of Syk and Btk.

Keywords: PIR-B; ITIM; SHP-1; SHP-2; tyrosine phosphorylation

\section{Introduction}

Antigen-mediated clustering of $\mathrm{B}$ cell antigen receptor (BCR) induces a transient protein tyrosine kinase (PTK) activity that activates several biochemical pathways and culminates in proliferation, differentiation, or apoptosis. Three distinct types of PTKs have been found to be activated upon BCR engagement: Src-family PTKs (including Lyn, Fyn, and Blk), Syk, and Btk. A multistep model for BCR signaling has been proposed in which Lyn is the first to be activated after BCR stimulation and phosphorylates the tyrosine residues in the ITAM sequences (for immunoreceptor tyrosine-based activation motif) within $\operatorname{Ig} \alpha$ and $\operatorname{Ig} \beta$ subunits. The phosphorylated ITAMs recruit Syk, which becomes phosphorylated and activated. Critical downstream signaling events include the activation of

*Correspondence: T Kurosaki

Received 9 September 1998; revised 21 October 1998; accepted 3 November 1998 phospholipase C (PLC)- $\gamma 2$, which is mediated by Syk and Btk. The activated PLC- $\gamma 2$ converts phosphatidylinositol 4,5-bisphosphate into the two second messengers diacylglycerol (DG) and inositol 1,4,5trisphosphate $\left(\mathrm{IP}_{3}\right)$ which results in activation of protein kinase $\mathrm{C}$ and increases in $\left[\mathrm{Ca}^{2+}\right]_{i}$, respectively (for reviews see references: DeFranco, 1997; Kurosaki, 1997; Pleiman et al., 1994; Reth and Wienands, 1997).

B cells express paired immunoglobulin-like receptor B (PIR-B), an integral membrane protein that contains six Ig-like domains in the extracellular portion and four potential ITIMs (for immunoreceptor tyrosinebased inhibitory motifs) in the cytoplasmic domain (Hayami et al., 1997; Kubagawa et al., 1997). We and others have recently demonstrated that PIR-B inhibits the BCR signaling in a phosphotyrosine-dependent manner and that, among four potential ITIMs, Tyr ${ }^{77}$ and $\mathrm{Tyr}^{801}$ are critical for mediating inhibitory signals (Figure 1) (Bléry et al., 1998; Maeda et al., 1998). Although the ligand for PIR-B is still unknown, PIR-B is inert until co-ligated to BCR at which time it undergoes tyrosine phosphorylation of ITIMs in its cytoplasmic domain. This phosphorylation leads to the recruitment of SHP-1 and SHP-2, which are responsible for the inhibition of calcium mobilization in the stimulated B cells.

In this report, we have examined the molecular mechanism underlying PIR-B-mediated inhibition of B cell activation. We first provide genetic evidence that Lyn is specifically required for tyrosine phosphorylation of critical ITIMs in the PIR-B cytoplasmic domain and its association with SHP-1 and SHP-2. The associated tyrosine phosphatases mediate dephosphorylation of Syk, Btk, and PLC- $\gamma 2$. Since activation of Syk and Btk is essential for PLC- $\gamma 2$ activation upon BCR engagement, our results suggest that downregulation of Syk and Btk acitivity by SHP-1 and SHP-2 is a potential mechanism for inhibition of $\mathrm{IP}_{3}$ generation and calcium mobilization by PIR-B.

\section{Results}

Recruitment of SHP-1 and SHP-2 to PIR-B ITIMs

Chimeric receptor Fc $\gamma$ RIIB-PIR-B with the cytoplasmic domain of PIR-B and the extracellular domain of mouse Fc $\gamma$ RIIB inhibits activation of B cells and mast cells like native PIR-B (Bléry et al., 1998; Maeda et al., 1998). Previous studies have demonstrated that among four potential ITIMs of PIR-B cytoplasmic domain (legends 
A

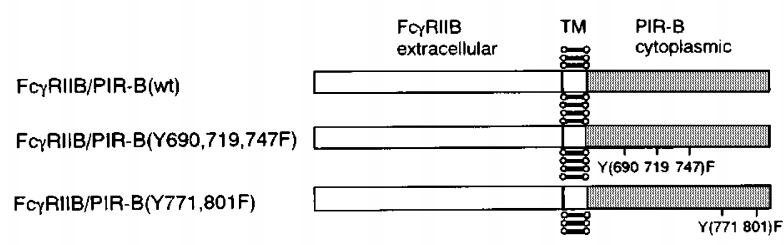

B

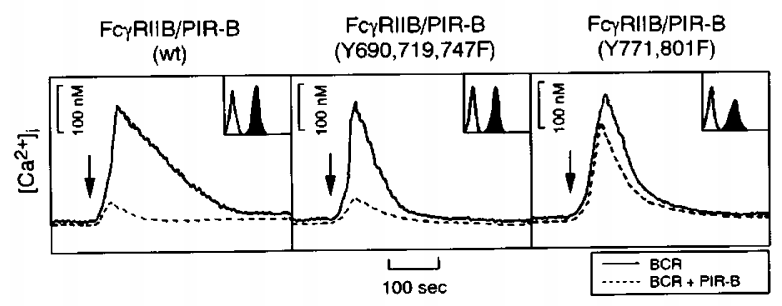

C

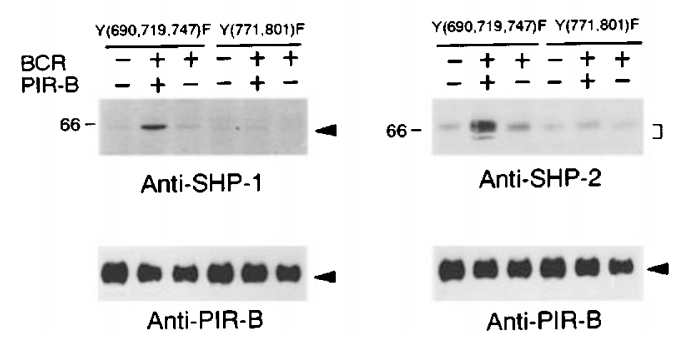

Figure $1 \mathrm{Tyr}^{771}$ and $\mathrm{Tyr}^{810}$ in PIR-B are required for $\mathrm{Fc} \gamma \mathrm{RIIB} /$ PIR-B-mediated inhibition of calcium mobilization and for recruitment of SHP-1 and SHP-2. (a) Schematic diagram of Fc $\gamma$ RIIB/PIR-B chimeric molecules. PIR-B cytoplasmic region contains four potential ITIMs (residues 688-693, SLYASV; 717-722, ETYAQV; 769-774, VTYAQL; and 799-804, SVYATL). In addition to these tyrosines, the cytoplasmic domain of PIR-B contains one more tyrosine (residue 747). The open and shaded boxes represent portions of Fc $\gamma$ RIIB and PIR-B, respectively. (b) Calcium mobilization stimulated by BCR crosslinking (solid line) and coligation of BCR to Fc $\gamma$ RIIB/PIR$\mathrm{B}$ (dashed line) in IIA1.6 cells expressing wild type and mutant Fc $\gamma$ RIIB/PIR-B. Histogram in the inserted box indicates the expression level of Fc $\gamma$ RIIB/PIR-B by $2.4 \mathrm{G} 2$ staining. Unstained cells were used as a negative control (open histogram). Stimulation of Fc $\gamma$ RIIB/PIR-B alone by $2.4 \mathrm{G} 2$ did not induce calcium mobilization. (c) Immunoblotting with anti-SHP-1 (left panel) and anti-SHP-2 (right panel) Abs after BCR ligation alone and the coligation of BCR to mutant Fc $\gamma$ RIIB/PIR-B. IIA1.6 cells expressing mutant Fc $\gamma$ RIIB/PIR-B stimulated for 3 min with $\mathrm{F}\left(\mathrm{ab}^{\prime}\right)_{2}$ rabbit anti-mouse IgG $(25 \mu \mathrm{g})$, or with intact rabbit antimouse IgG $(50 \mu \mathrm{g})$ were lysed by NP-40 buffer. Fc $\gamma$ RIIB/PIR-B was then immunoprecipitated with $2.4 \mathrm{G} 2$, resolved by SDSPAGE gel, transferred to membrane, and probed with anti-SHP-1 $\mathrm{Ab}$ or anti-SHP-2 Ab. Membranes were reprobed with anti-PIR$\mathrm{B} \mathrm{Ab}$ (lower panel)

in Figure 1a), phosphorylation of both $\mathrm{Tyr}^{771}$ and $\mathrm{Tyr}^{801}$ is involved in PIR-B-mediated inhibitory signal presumably through recruitment of tyrosine phosphatases SHP-1 and SHP-2 (Bléry et al., 1998; Maeda et al., 1998). To formally demonstrate that the PIR-B cytoplasmic region containing doubly phosphorylated $\mathrm{Tyr}^{771}$ and $\mathrm{Tyr}^{801}$ is indeed the recruitment site for SHP-1 and SHP-2 in vivo, we transfected Fc $\gamma$ RIIB-PIR-B mutant in which all remaining Tyr residues in the PIR-B cytoplasmic domain are changed to Phe (Y690, 719, 747F), into the Fc $\gamma$ RIIB-negative B cell mutant cell line (A20 IIA1.6) (Figure 1a). Cell surface expression level of this chimeric receptor was checked by flow cytometric analysis using anti-mouse Fc $\gamma$ RIIB mAb, $2.4 \mathrm{G} 2$ (shown in Figure $1 \mathrm{~b}$ inset box). As demonstrated in Figure $1 \mathrm{~b}$ and $\mathrm{c}$, coligation of Fc $\gamma$ RIIB-PIR-B(Y690, 719, 747F) and $\mathrm{BCR}$ inhibited the calcium mobilization and recruited SHP-1 and SHP-2. Conversely, Fc $\gamma$ RIIB-PIR-B(Y771, $810 \mathrm{~F}$ ) neither inhibited the BCR-induced calcium mobilization nor recruited SHP-1 and SHP-2. Three species of SHP-2 which migrated distinctly (right panel in Figure 1c) are presumably due to post-translational and/ or post-transcriptional modifications of SHP-2, because three SHP-2 species similarly observed in DT40 B cells could not be detected after targeted deletion of the SHP2 gene (data not shown).

Lyn is required for tyrosine phosphorylation of PIR-B at $T^{7 y} r^{771}$ and $T y r^{801}$

The above observations prompted us to examine the responsible PTK(s) for Fc $\gamma$ RIIB-PIR-B(Y690, 719, $747 \mathrm{~F})$ tyrosine phosphorylation upon its coligation with BCR. For this purpose, we trasnfected Fc $\gamma$ RIIBPIR-B(Y690, 719, 747F) into Lyn- or Syk-deficient mutant DT40 cells (Takata et al., 1994). After obtaining transformants expressing similar level of Fc $\gamma$ RIIB-PIR-B(Y690, 719, 747F), DT40 cells deficient in Lyn or Syk were stimulated by BCR crosslinking alone or coligation of BCR and the inhibitory receptor (Figure 2). Consistent with our previous results using mouse A20 B cells (Maeda et al., 1998), the chimeric receptor was slightly tyrosine phosphorylated upon BCR cross-linking alone and this phosphorylation was markedly augumented by coligation of BCR and Fc $\gamma$ RIIB-PIR-B (Y690, 719, 747F) in wild type DT40 B cells. Syk-deficient DT40 cells showed the similar phosphorylation status of Fc $\gamma$ RIIB-PIR$\mathrm{B}(\mathrm{Y} 690,719,747 \mathrm{~F})$ to wild type cells, whereas this Fc $\gamma$ RIIB-PIR-B(Y690, 719, 747F) phosphorylation was abrogated by loss of Lyn. These results demonstrate that Lyn is required for phosphorylation of $\mathrm{Tyr}^{771}$ and $\mathrm{Tyr}^{801}$ in the PIR-B cytoplasmic domain.

\section{PIR-B inhibits PLC- $\gamma 2$ activation}

$\mathrm{IP}_{3}$ generation by PLC- $\gamma 2$ activation is essential for BCR-induced calcium mobilization from intracellular pools (Sugawara et al., 1997; Takata et al., 1995). Then, $\mathrm{Ca}^{2+}$ enters the cytoplasm by a process termed capacitative $\mathrm{Ca}^{2+}$ entry, by which the depletion of intracellular stores by $\mathrm{IP}_{3}$ activates calcium influx across the plasma membrane (Putney and Bird, 1993). Thus, inhibition of the BCR-induced calcium mobilization by PIR-B could be accounted for by two possible mechanisms: inhibition of the BCR-induced $\mathrm{IP}_{3}$ generation or inhibition of capacitative $\mathrm{Ca}^{2+}$ entry process. To distinguish these two possibilities, we determined $\mathrm{IP}_{3}$ generation by $\mathrm{BCR}$ cross-linking alone or coligation of BCR and Fc $\gamma$ RIIB-PIR-B. As shown in Figure 3a, BCR cross-linking alone generated $\mathrm{IP}_{3}$ while this generation was abrogated by coligation of the inhibitory receptor and BCR. Consistent with this result, BCR-induced tyrosine phosphorylation of PLC$\gamma 2$ was profoundly inhibited by its coligation with Fc $\gamma$ RIIB-PIR-B (Figure 3b). Thus, we conclude that coligation of BCR and Fc $\gamma$ RIIB-PIR-B inhibits PLC$\gamma 2$ tyrosine phosphorylation, thereby leading to inhibition of $\mathrm{IP}_{3}$ generation. 


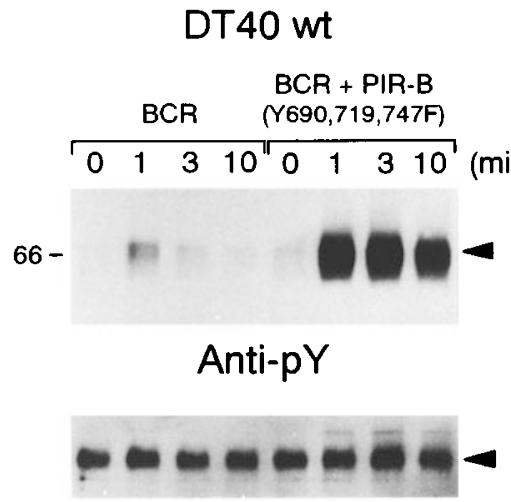

Anti-PIR-B
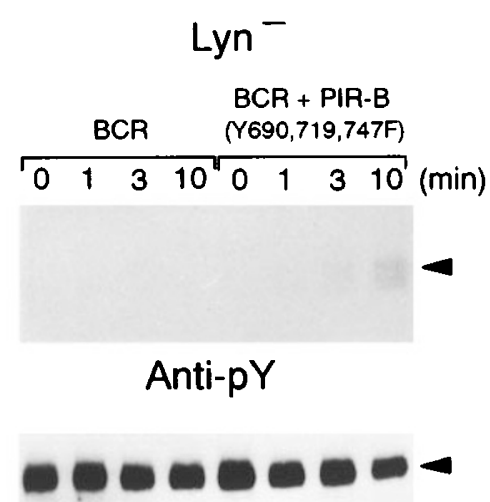

Anti-PIR-B

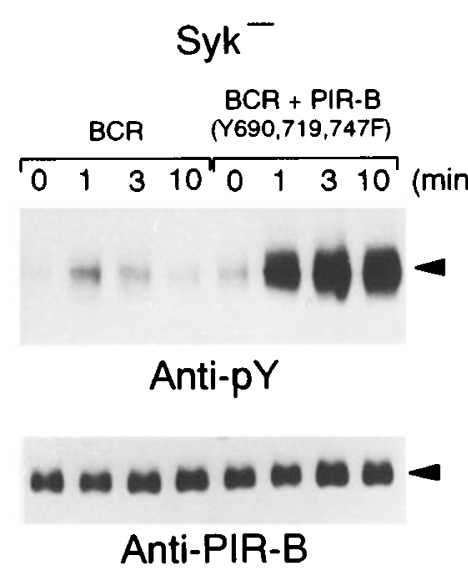

Figure 2 Tyrosine phosphorylation of Fc $\gamma$ RIIB/PIR-B(Y690, 719, 747F) by BCR crosslinking alone and coligation in wildtype, Lyn-negative, and Syk-negative DT40 cells. The cells expressing Fc $\gamma$ RIIB/PIR-B(Y690, 719, 747F) were incubated with F(ab') rabbit anti-mouse $\operatorname{IgM}(10 \mu \mathrm{g})$, or with intact rabbit anti-mouse IgM $(20 \mu \mathrm{g})$, then stimulated by anti-chicken IgM mAb M4 (4 $\mu \mathrm{g})$. Fc $\gamma$ RIIB/PIR-B (Y690, 719, 747F) was immunoprecipitated with 2.4G2, separated by SDS-PAGE gel, transferred to membrane, and immunoblotted with anti-phosphotyrosine mAb 4G10 (upper panel). The same membrane was reprobed with anti-PIR-B Ab (lower panel)

\section{Syk and Btk are potential targets of SHP-1}

Early activation of PTKs, particularly Syk and Btk, is involved in PLC- $\gamma 2$ activation upon BCR engagement (Kurosaki, 1997). Therefore, data presented in Figure 3 could be explained by PIR-B-mediated dephosphorylation of PLC- $\gamma 2$ and/or its upstream PTKs, Syk and Btk. To examine these possibilities, the status of tyrosine phosphorylation of Syk and Btk was determined by immunoprecipitation followed by Western blotting with antiphosphotyrosine mAb. As shown in Figure $4 \mathrm{a}$ and $\mathrm{b}$, the BCR-induced tyrosine phosphorylation of Syk and Btk was dramatically inhibited by coligation of BCR and Fc $\gamma$ RIIB-PIR-B. Moreover, dephosphorylation of Syk and Btk by Fc $\gamma$ RIIB-PIR-B resulted in inhibition of their kinase activity, as assessed by in vitro kinase assay. As reported previously (Burkhardt et al., 1991; Yamanashi et al., 1992), Lyn is activated about 1.5-fold upon BCR stimulation alone (Figure 4c). In contrast to inhibition of Syk and Btk, coligation of the BCR and Fc $\gamma$ RIIB-PIR-B did not inhibit the tyrosine phosphorylation and kinase activity of Lyn. Since binding of Syk to phosphorylated $\operatorname{Ig} \alpha / \operatorname{Ig} \beta$ is essential for participation of Syk in downstream signaling events such as PLC- $\gamma 2$ activation (DeFranco, 1997; Kurosaki, 1997; Pleiman et al., 1994; Reth and Wienands, 1997), we also determined whether BCR-induced tyrosine phosphorylation of $\operatorname{Ig} \alpha$ and $\operatorname{Ig} \beta$ is inhibited by its coligation with $\mathrm{Fc} \gamma \mathrm{RIIB}-\mathrm{PIR}-\mathrm{B}$. As shown in Figure $4 \mathrm{~d}$, coligation of the BCR and Fc $\gamma$ RIIB-PIR-B inhibited phosphorylation of both $\operatorname{Ig} \alpha$ and $\operatorname{Ig} \beta$. Thus, these observations indicate that ligation of Fc $\gamma$ RIIBPIR-B inhibits BCR-induced tyrosine phosphorylation of four critical signaling molecules: $\operatorname{Ig} \alpha / \operatorname{Ig} \beta$, Syk, Btk, and PLC- $\gamma 2$. Furthermore, dephosphorylation of Syk and Btk leads to inhibition of their kinase activity.

To investigate whether the Fc $\gamma$ RIIB-PIR-B-mediated inhibition of tyrosine phosphorylation of signaling molecules could be attributed to SHP-1, we employed vaccinia virus-driven overexpression system of SHP-1 (Figure 5). Expression of the catalytically inactive
SHP-1-C453S, but not wild type SHP-1, significantly reversed the Fc $\gamma$ RIIB-PIR-B-mediated inhibition of tyrosine phosphorylation of Syk, Btk, and PLC- $\gamma 2$ (Figure 6). Moreover, kinase activity of Syk and Btk was also restored by expression of SHP-1-C453S. In contrast, inhibition of $\operatorname{Ig} \alpha$ tyrosine phosphorylation was less reversed than Syk and Btk. These results demonstrate that Fc $\gamma$ RIIB-PIR-B ligation interrupts tyrosine phosphorylation of Syk, Btk, and PLC- $\gamma 2$ via an SHP-1 dependent mechanism.

\section{Discussion}

Recent studies have shown that among the five tyrosine residues in the PIR-B cytoplasmic domain, phosphorylation of $\mathrm{Tyr}^{771}$ and $\mathrm{Tyr}^{801}$ is required for its inhibitory mechanism (Bléry et al., 1998; Maeda et al., 1998). Here we extended these data to show that phosphorylation of remaining Tyr residues $\left(\mathrm{Tyr}^{690}, \mathrm{Tyr}^{719}, \mathrm{Tyr}^{747}\right)$ is dispensable for PIR-B-mediated inhibition and that SHP-1 and SHP-2 are recruited to the PIR-B Tyr ${ }^{771}$ and $\mathrm{Tyr}^{801}$ ITIMs in vivo. This observation, together with the evidence that both SHP-1 and SHP-2 are able to bind to the phosphorylated ITIM peptide including $\mathrm{Tyr}^{771}$ or $\mathrm{Tyr}^{801}$ in vitro (Bléry et al., 1998), indicates that association of SHP-1 and SHP-2 to the PIR-B $\mathrm{Tyr}^{771}$ and $\mathrm{Tyr}^{801}$ ITIMs is mediated via an SH2phosphotyrosine interaction.

Data presented here demonstrate that Lyn, but not Syk, is required for PIR-B ITIM tyrosine phosphorylation in DT40 B cells. Because Lyn is dominantly expressed among Src-family PTKs particularly in DT40 cells (Takata et al., 1994), it is likely that other Srcfamily PTKs such as Fyn and Blk are also capable of phosphorylating PIR-B ITIMs. Since Lyn is apparently involved in activating BCR signal by phosphorylating ITAMs in $\operatorname{Ig} \alpha / \operatorname{Ig} \beta$ and Syk (DeFranco, 1997; Kurosaki, 1997; Pleiman et al., 1994; Reth and Wienands, 1997), our results support the contention that Lyn has both positive and negative roles in B cell activation (DeFranco, 1997; Kurosaki, 1997). The 
A

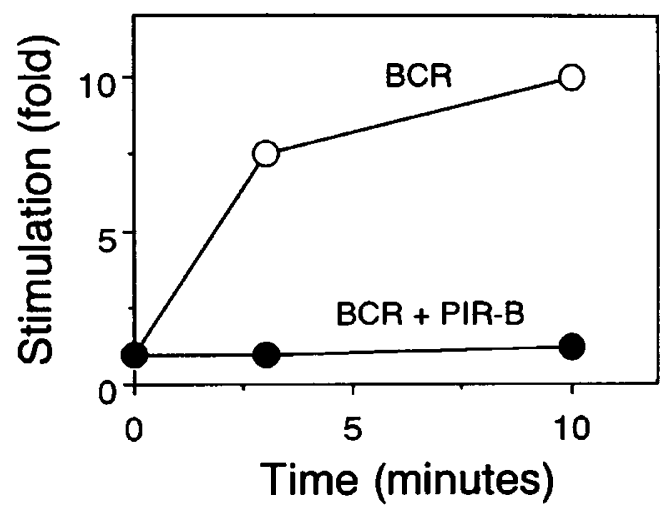

B

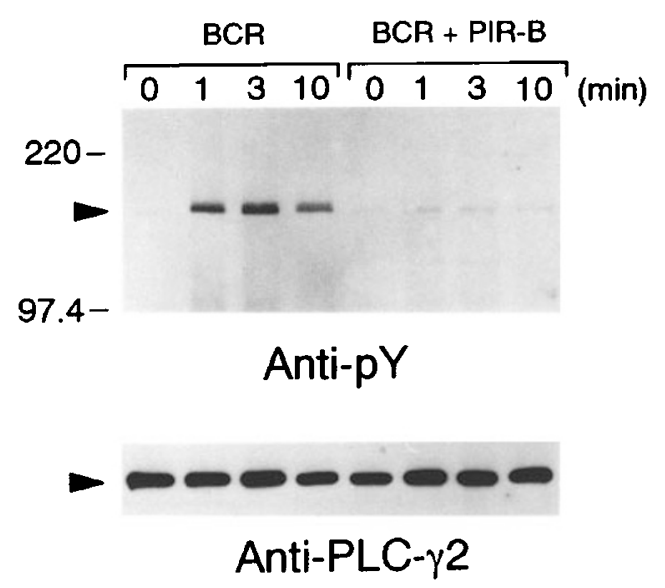

Figure 3 Fc $\gamma$ RIIB/PIR-B-mediated inhibitory effect on PLC- $\gamma 2$. (a) Phosphoinositide hydrolysis after BCR crosslinking alone and coligation. IIA1.6 cells expressing wild type Fc $\gamma$ RIIB/PIR-B were stimulated with $\mathrm{F}\left(\mathrm{ab}^{\prime}\right)_{2}$ rabbit anti-mouse $\operatorname{IgG}(10 \mu \mathrm{g})$, or with intact rabbit anti-mouse $\operatorname{IgG}(20 \mu \mathrm{g})$. Soluble inositol was extracted and separated by the AG 1-X8 ion exchange columns. (b) Tyrosine phosphorylation of PLC- $\gamma 2$. After stimulation, PLC$\gamma 2$ was immunoprecipitated with anti-PLC- $\gamma 2$ serum, separated by SDS-PAGE gel, transferred to membrane, and immunoblotted with anti-phosphotyrosine mAb 4G10 (upper panel). The same membrane was reprobed with anti-PLC- $\gamma 2$ serum (lower panel)

negative role of Lyn appears to be mediated in part by PIR-B as well as FcyRIIB and CD22 (Chan et al., 1997; Nishizumi et al., 1998; O'Rourke et al., 1997; Wang et al., 1996). Slight PIR-B tyrosine phosphorylation by BCR alone, although less efficient than occurring after coligation of BCR and PIR-B (Figure 2 ), might provide insights into the mechanism by which PIR-B modulates $\mathrm{B}$ cell activation in vivo. Assuming that a small fraction of SHP-1 or SHP-2 is recruited to the slightly phosphorylated PIR-B by BCR ligation alone, a small portion of the inhibitory function of PIR-B might not necessarily require coligation of $\mathrm{BCR}$ and PIR-B. Nevertheless, it is clear that coligation of BCR and PIR-B inhibits BCR activation more dramatically.

Here we explored the molecular mechanisms of PIRB-mediated inhibition of $\mathrm{B}$ cell activation. In contrast to FcrRIIB inhibition (Gupta et al., 1997; Hippen et
A

B

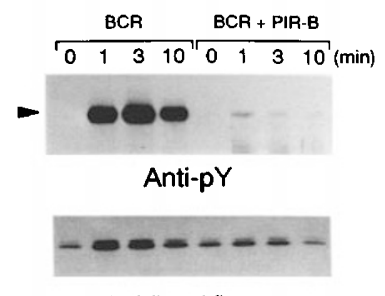

In Vitro Kinase

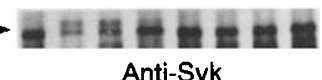

C

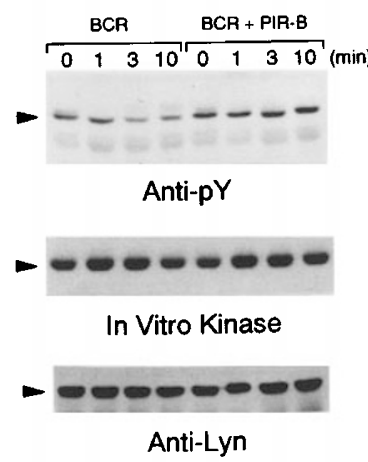

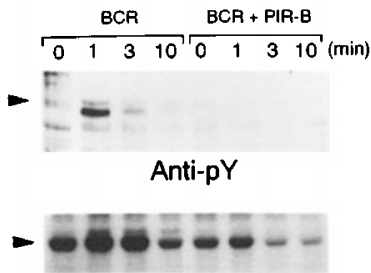

In Vitro Kinase

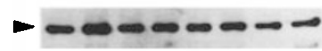
Anti-Btk
D

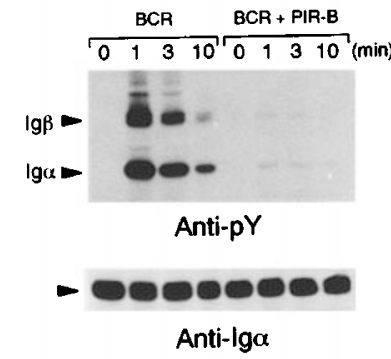

Figure 4 Fc $\gamma$ RIIB/PIR-B-mediated inhibition on Syk, Btk, and $\operatorname{Ig} \alpha$. Tyrosine phosphorylation of Syk, Btk, Lyn, and $\operatorname{Ig} \alpha(\mathbf{a}-\mathbf{d})$ and in vitro kinase activity of Syk (middle panel in a), Btk (middle panel in b), and Lyn (middle panel in c). After stimulation, IIA1.6 cells expressing wild type FcrRIIB/PIR-B were lysed and immunoprecipitated with anti-Syk (a), anti-Btk (b), anti-Lyn (c), or anti-Ig $\alpha$ (d). As shown previously (Kurosaki et al., 1994), another slower migrating Syk species was observed upon BCR stimulation. Immunoprecipitates were divided, and half of them were used for Western blotting with mAb 4G10 (upper panel). After the filter was stripped, the same blot was reprobed (lower panel). The remaining half were used for in vitro kinase assay (middle panel in $\mathbf{a}-\mathbf{c}$ ). Reaction samples in Syk were separated by $12.5 \%$ SDS-PAGE gel, transferred and immunoblotted with anti-phosphotyrosine mAb 4G10. For Btk and Lyn, samples were electrophoresed by $8 \%$ SDS-PAGE gel

al., 1997; Nadler et al., 1997; Ono et al., 1997), BCRinduced activation of the upstream tyrosine kinases Syk and Btk, as well as many other substrates were drastically inhibited by its coligation with PIR-B, strengthening our previous conclusion that PIR-B inhibits the $\mathrm{B}$ cell activation by utilizing a different inhibitory mechanism than Fc $\gamma$ RIIB. Thus, PIR-B apparently belongs to a class of KIR-type inhibitory receptors (Ono et al., 1997). Indeed, similar to PIR-Bmediated inhibition, coligation of FcR and KIR inhibits tyrosine phosphorylation of many of substrate phosphorylated by stimulation of the FcR alone (Binstadt et al., 1996).

Since $\mathrm{IP}_{3}$ generation and its binding to $\mathrm{IP}_{3}$ receptors are essential for the BCR-induced calcium mobilization from intracellular pools (Sugawara et al., 1997; Takata et al., 1995), our data indicate that PIR-B aborts the initial release of $\mathrm{Ca}^{2+}$ from the intracellular pools by inhibiting $\mathrm{IP}_{3}$ generation through down-regulation of PLC- $\gamma 2$. As SHP-1 and SHP-2 are required for PIR-B- 


\section{SHP-1}

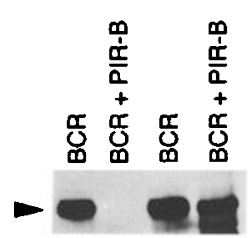

$\mathrm{C} 453 \mathrm{~S}$
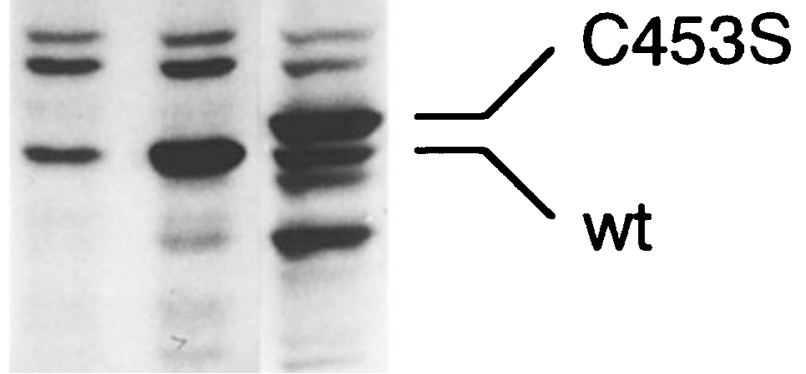

Figure 5 Expression of wild type SHP-1 and SHP-1C453S. Whole cell extracts from cells infected with vector virus (V) or recombinant virus encoding wild type SHP-1 (wt) and SHP-1 C453S (CS), were subject to SDS-PAGE gel, followed by Western blot analysis with anti-SHP-1

mediated inhibition of calcium mobilization (Maeda et al., 1998), the simplest interpretation for inhibition of PLC $-\gamma 2$ tyrosine phosphorylation is that PLC $-\gamma 2$ serves as a direct substrate of SHP-1 and SHP-2. Assuming that PLC $-\gamma 2$ is a sole substrate of SHP-1 and SHP-2 by PIR-B, one would predict that engagement of PIR-B with BCR dephosphorylates only PLC- $\gamma 2$ rather than many substrates. However, as shown in this study, not only PLC- $\gamma 2$ but also several signaling molecules (Figure 4) were dephosphorylated by coligation of PIR-B and BCR. Furthermore, analyses of phosphotyrosine-containing proteins of whole cellular lysates indicate the inhibition of tyrosine phosphorylation of many proteins by PIR-B (data not shown). Thus, our data favor a model in which the activation of upstream PTKs rather than PLC- $\gamma 2$ itself are down-modulated by PIR-B ligation.

Among BCR-activated PTKs, Syk and Btk are particularly important, since genetic studies indicate the requirement of Syk and Btk for BCR-induced tyrosine phosphorylation of PLC- $\gamma 2$ (Fluckiger et al., 1998; Scharenberg et al., 1998; Takata and Kurosaki, 1996; Takata et al., 1994). Indeed, BCR-induced activation of Syk and Btk was profoudly inhibited by coligation of PIR-B and BCR, and this inhibition was substantially recovered by overexpression of catalytically inactive SHP-1-C453S. As PIR-B-mediated inhibition requires redundant function of SHP-1 and SHP-2 (Maeda et al., 1998), overexpression of SHP1C-453S would likely block the function of both SHP-1 and SHP-2 to a similar extent. PIR-B-mediated dephosphorylation of $\operatorname{Ig} \alpha$ and $\operatorname{Ig} \beta$ could also account for down-modulation of Syk, because tyrosine phosphorylation of Syk is followed by its association with phosphorylated $\operatorname{Ig} \alpha / \operatorname{Ig} \beta$ (DeFranco, 1997; Kurosaki, 1997; Pleiman et al., 1994; Reth and Wienands, 1997). In contrast to substantial restoration of Syk and Btk phosphorylation by SHP-1-C453S, less restoration of $\operatorname{Ig} \alpha / \operatorname{Ig} \beta$ phosphorylation suggests that PIR-B-

In Vitro Kinase
A

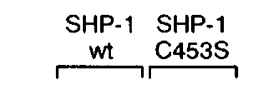

B
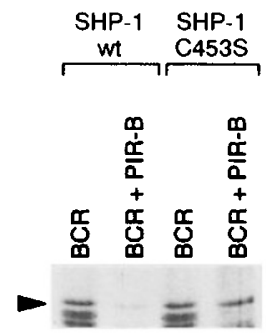

Anti-pY
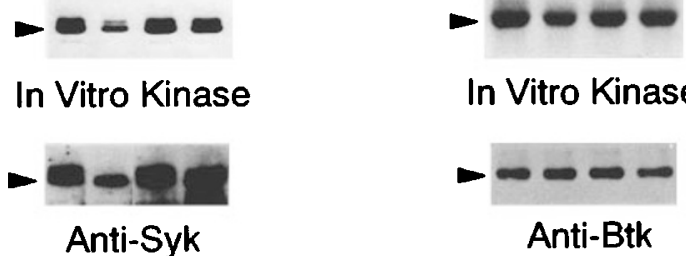

Anti-Btk

C

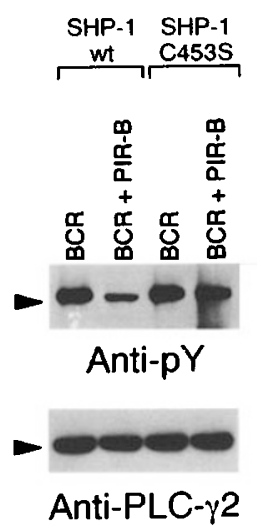

D
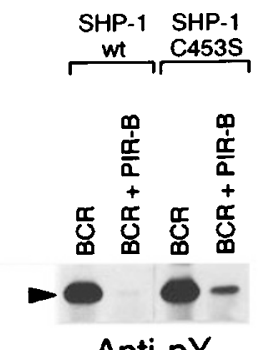

Anti-pY

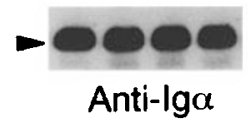

Figure 6 Restoration of Fc $\gamma$ RIIB/PIR-B-mediated inhibitory effects by overexpression of catalytically inactive SHP-1. Tyrosine phosphorylation of Syk, Btk, PLC- $\gamma 2$, and $\operatorname{Ig} \alpha(\mathbf{a}-\mathbf{d})$ and in vitro kinase activity of Syk (middle panel in a) and Btk (middle panel in b). IIA1.6 cells expressing wild type Fc $\gamma$ RIIB/ PIR-B $\left(5 \times 10^{6}\right.$ per lane $)$ were infected with recombinant vaccinia encoding either wild type SHP-1 or catalytically inactive SHP-1C453S. After stimulation (1 min), cells were lysed and immunoprecipitated with anti-Syk (a), anti-Btk (b), anti-PLC- $\gamma 2$ (c) or anti-Ig $\alpha$ (d). Immunoprecipitates were immunoblotted with $\mathrm{mAb} 4 \mathrm{G} 10$ (upper panel). After the filter was stripped, the same blot was reprobed (lower panel). Immunoprecipitates with antiSyk or anti-Btk were divided, and half of them were used for Western blotting. The remaining half were used for in vitro kinase assay (middle panel in $\mathbf{a}$ and $\mathbf{b}$ )

mediated $\operatorname{Ig} \alpha / \operatorname{Ig} \beta$ dephosphorylation is mediated by another type of tyrosine phosphtase(s). Involvement of this phosphatase, in addition to SHP-1 and SHP-2, might explain the residual PIR-B inhibition in SHP-1 and SHP-2 double-deficient DT40 cells (Maeda et al., 1998). In contrast to Syk and Btk, Lyn activation was not significantly inhibited by PIR-B ligation. Thus, our data indicate that among the three types of BCRactivated PTKs, Syk and Btk are specifically downmodulated by SHP-1 and SHP-2, thereby leading to inhibition of PLC- $\gamma 2$ phosphorylation.

In summary, our data support the following mechanism: (a) Coligation of PIR-B and BCR results in phosphorylation of PIR-B ITIMs by Lyn; (b) SHP-1 and SHP-2 are recruited to the phosphorylated PIR-B ITIMs via their SH2 domains, and are subsequently 
activated; (c) activated SHP-1/SHP-2 dephosphorylate their substrates apparently including Syk and Btk; (d) down-modulation of Syk and Btk by presumably dephosphorylation of tyrosine residues in their activation loop inhibits PLC- $\gamma 2$ activation; (e) downmodulated PLC- $\gamma 2$ decreases $\mathrm{IP}_{3}$ generation, leading to a block of calcium mobilization.

\section{Materials and methods}

\section{Cells, expression construct and Abs}

Various mutant DT40 cells and A20 IIA1.6 cells were maintained in RPMI 1640 supplemented with 10\% FCS, penicillin, streptomycin, and glutamine. For making Fc $\gamma$ RIIB/PIR-B chimera, the cDNA of mouse Fc $\gamma$ RIIB (corresponding to amino acid residue: -29 to 207 including signal peptide) and PIR-B (corresponding to amino acid residue: $641-818$ ) were fused by using PCR. Mutations that tyrosine residues at posistions 690, 719, 747, 771 and 801 to phenylalanine were introduced by the PCR method. Resulting constructs were confirmed by DNA sequencing. The mutant and wild type Fc $\gamma$ RIIB/ PIR-B cDNAs were subcloned into pApuro vector (Takata et al., 1994) and were electroporated into DT40 or A20 IIA1.6 cells as described previously (Sugawara et al., 1997). After selecting clones in the presence of puromycin $(0.5 \mu \mathrm{g} / \mathrm{ml})$, cell surface expression levels of Fc $\gamma$ RIIB/PIR-B were checked by flow cytometry analysis using anti-mouse $\mathrm{Fc} \gamma \mathrm{RIIB} \mathrm{mAb}, 2.4 \mathrm{G} 2$ (Unkeless, 1979). Anti-chicken IgM mAb M4, anti-Syk Ab, anti-PLC- $\gamma 2$ $\mathrm{Ab}$, anti-SHP-1 Ab, anti-Ig $\alpha$ Ab were already described (Maeda et al., 1998; Sanchez et al., 1993; Takata et al., 1994). Anti-PIR-B Ab was obtained by immunizing rabbits with bacterially expressed glutathione S-transferase fusion protein containing PIR-B cytoplasmic region. Anti-SHP-2 Ab, intact rabbit anti-mouse IgM, $\mathrm{F}\left(\mathrm{ab}^{\prime}\right)_{2}$ rabbit anti-mouse $\operatorname{IgM}$, intact rabbit anti-mouse $\mathrm{IgG}$, $\mathrm{F}\left(\mathrm{ab}^{\prime}\right)_{2}$ rabbit anti-mouse $\mathrm{IgG}$, and anti-phosphotyrosine $\mathrm{mAb} 4 \mathrm{G} 10$ were purchased from Santa Cruz Biotechnology Inc, Zymed laboratories Inc, Chemicon Inc, Chemicon Inc, and Upstate Biotechnology Inc, respectively. The anti-Btk mAb 43-3B was used for immunoprecipitation and Western blotting of Btk (Baba et al., in press). Polyclonal anti-Lyn $\mathrm{Ab}$ used to immunoprecipitate was kindly provided by Drs $\mathrm{H}$ Umemori and $\mathrm{T}$ Yamamoto (Institute of Medical Science, University of Tokyo., unpublished data). Western blotting of Lyn was accomplished using the monoclonal anti-Lyn, Lyn 8 (Yamanashi et al., 1992).

\section{Vaccinia viruses}

Recombinant vaccinia viruses encoding wild type and dominant-negative forms (cysteine 453 to serine) of SHP1 have been described (Burshtyn et al., 1996). For infections, A20 IIA1.6 cells $\left(2 \times 10^{6}\right.$ cells $\left./ \mathrm{ml}\right)$ were incubated in serum-free RPMI 1640 for $1 \mathrm{~h}$ at $37^{\circ} \mathrm{C}$ at a multiplicity of infection of 20. Cells were then incubated for $3 \mathrm{~h}$ at $10^{6}$ cells/ml in RPMI 1640 supplemented with $10 \%$ FCS.

\section{Immunoprecipitation and Western blotting analysis}

Cells were solubilized in lysis buffer (1\% NP-40, $150 \mathrm{~mm}$ $\mathrm{NaCl}, 20 \mathrm{~mm}$ Tris, pH 7.5, $1 \mathrm{~mm}$ EDTA, $10 \%$ glycerol) containing $50 \mathrm{~mm} \mathrm{NaF}, 10 \mu \mathrm{M}$ molybdate, $2 \mathrm{~mm}$ sodium vanadate supplemented with protease inhibitors as described previously (Sugawara et al., 1997). For immunoprecipitation, precleared lysates were sequentially incubated with Abs and protein A-agarose or anti-rat IgG- agarose. Lysates or immunoprecipitates were separated by SDS-PAGE gel, transferred to nitrocellulose membrane, and detected by appropriate Abs and ECL system (Amersham Corp.). To examine in vitro kinase activity of Syk, immunoprecipitated Syk was suspended in kinase buffer $\left(20 \mathrm{~mm}\right.$ Tris, $\mathrm{pH} 7.5,10 \mathrm{mM} \mathrm{MgCl}_{2}, 10 \mathrm{mM} \mathrm{MnCl}$ ) in the presence of ATP $(1 \mu \mathrm{M})$. Recombinant glutathione $S$-transferase fusion protein containing a cytoplasmic domain of mouse $\operatorname{Ig} \alpha(5 \mu \mathrm{g})$ was used as an exogenous substrate (Saouaf et al., 1994). The reactions were allowed at $30^{\circ} \mathrm{C}$ for $10 \mathrm{~min}$ and terminated by the addition of sample buffer. The mixture was separated by SDS-PAGE gel, transferred to nitrocellulose membrane, and detected by $4 \mathrm{G} 10$ and ECL system. For in vitro kinase assay of Lyn and Btk, immunoprecipitates were suspended in kinase buffer $\left(20 \mathrm{mM}\right.$ PIPES, pH 7.5, $20 \mathrm{mM} \mathrm{MnCl}_{2}$ ) containing $\left[\gamma-{ }^{32} \mathrm{P}\right]$ ATP $(>3000 \mathrm{Ci} / \mathrm{mmol}$, Amersham), $5 \mu \mathrm{g}$ of enolase (Sigma) and incubated at $25^{\circ} \mathrm{C}$ for $3 \mathrm{~min}$. The reaction was stopped by addition of sample buffer. The mixture was separated by SDS-PAGE gel, followed by autoradiography.

\section{Calcium and phosphoinositide analysis}

For calcium analysis, cells $\left(5 \times 10^{6}\right)$ were suspended in PBS containing $20 \mathrm{mM}$ HEPES (pH 7.2), $5 \mathrm{~mm}$ glucose, $0.025 \%$ BSA and $1 \mathrm{mM} \mathrm{CaCl}_{2}$, and loaded with $3 \mu \mathrm{M}$ Fura-2/AM at $37^{\circ} \mathrm{C}$ for $45 \mathrm{~min}$. Cells were washed twice, and adjusted to $10^{6}$ cells $/ \mathrm{ml}$. Continuous monitoring of fluorescence from the cell suspension was performed using Hitachi F2000 fluorescence spectrophotometer (Hitachi Limited, Tokyo, Japan) at an excitation wavelength of $340 \mathrm{~nm}$ and an emission wavelength of $510 \mathrm{~nm}$. Calibration and calculation of calcium levels were done as described (Grynkiewicz et al., 1985). For phosphoinositide analysis, cells $\left(10^{6} / \mathrm{ml}\right)$ were labeled with myo- ${ }^{3} \mathrm{H}$-inositol $(10 \mu \mathrm{Ci} / \mathrm{ml}$, $105 \mathrm{Ci} / \mathrm{mmol}$, Amersham) for $5-6 \mathrm{~h}$ in inositol-free RPMI1640 supplemented with $10 \%$ dialyzed FCS, then stimulated with intact or $F\left(a b^{\prime}\right)_{2}$ fragment of rabbit antimouse IgG. The soluble inositol phosphates were extracted with trichloroacetic acid at indicated time points, and applied to AG 1-X8 (formate form) ion exchange columns (Bio-Rad Laboratories) pre-equilibrated with $0.1 \mathrm{M}$ formic acid. The columns were washed with $10 \mathrm{ml}$ water and $10 \mathrm{ml}$ of $60 \mathrm{~mm}$ ammonium formate $/ 5 \mathrm{~mm}$ sodium tetraborate. Elution was performed with increasing concentrations of ammonium formate $(0.1-0.7 \mathrm{M})$ (Berridge et al., 1983).

\section{Flow cytometric analysis for surface expression of FC $\gamma$ RIIB/PIR-B}

Cells were washed, stained with FITC-conjugated 2.4G2 (PharMingen) and analysed by FACScan (Becton-Dickinson \& Co.). The $x$ axes and $y$ axes for the histograms in inserted boxes (Figure 1b) indicate fluorescence intensity (4-decade-log scales) and relative cell number, respectively.

\section{Acknowledgements}

We would like to acknowledge $M$ Kurosaki for the technical assistance, Dr H Umemori and Dr T Yamamoto for providing anti-Lyn $\mathrm{Ab}$, and $\mathrm{Dr} \mathrm{S}$ Arai (Fujisaki institute, Hayashibara Biochemical Laboratories Inc.) for generation of anti-Btk mAb 43-3B. This work was supported by grants to TK from the Ministry of Education, Science, Sports, and Culture of Japan, the Uehara Memorial Foundation, and the Sumitomo Foundation. 


\section{References}

Baba Y, Nonoyama S, Matsushita M, Yamadori T, Hashimoto S, Imai K, Arai S, Kunikata T, Kurimoto M, Kurosaki T, Ochs HD, Yata J, Kishimoto $\mathrm{T}$ and Tsukata S. Blood, in press.

Berridge MJ, Dawson RMC, Downes CP, Heslop JP and Irvine RF. (1983). Biochem. J., 212, 473-482.

Binstadt BA, Brumbaugh KM, Dick CJ, Scharenberg AM, Williams BL, Colonna M, Lanier LL, Kinet J-P, Abraham RT and Leibson PJ. (1996). Immunity, 5, 629-638.

Bléry M, Kubagawa H, Chen C-C, Vély F, Cooper MD and Vivier E. (1998). Proc. Natl. Acad. Sci. USA, 95, $2446-$ 2451.

Burkhardt AL, Brunswick M, Bolen JB and Mond JJ. (1991). Proc. Natl. Acad. Sci. USA, 88, 7410-7414.

Burshtyn DN, Scharenberg AM, Wagtmann N, Rajagopalan S, Berrada K, Yi T, Kinet J-P and Long EO. (1996). Immunity, 4, 77-85.

Chan VWF, Meng F, Soriano P, DeFranco AL and Lowell CA. (1997). Immunity, 7, 69-81.

DeFranco AL. (1997). Curr. Opin. Immunol., 9, 296-308.

Fluckiger A-C, Li Z, Kato RM, Wahl MI, Ochs HD, Longnecker R, Kinet J-P, Witte ON, Scharenberg AM and Rawlings DJ. (1998). EMBO J., 17, 1973-1985.

Grynkiewicz G, Poenie M and Tsien RY. (1985). J. Biol. Chem., 260, 3440-3450.

Gupta N, Scharenberg AM, Burshtyn DN, Wagtmann N, Lioubin MN, Rohrschneider LR, Kinet J-P and Long EO. (1997). J. Exp. Med., 186, 473-478.

Hayami K, Fukuta D, Nishikawa Y, Yamashita Y, Inui M, Ohyama Y, Hikita M, Ohmori H and Takai T. (1997). J Biol. Chem., 272, 7320-7327.

Hippen KL, Buhl AM, D’Ambrosio D, Nakamura K, Persin C and Cambier JC. (1997). Immunity, 7, 49-58.

Kubagawa H, Burrows PD and Cooper MD. (1997). Proc. Natl. Acad. Sci. USA, 94, $5261-5266$.

Kurosaki T. (1997). Curr. Opin. Immunol., 9, 309-318.

Kurosaki T, Takata M, Yamanashi Y, Inazu T, Taniguchi T, Yamamoto T and Yamamura H. (1994). J. Exp. Med., 179, $1725-1729$.
Maeda A, Kurosaki M, Ono M, Takai T and Kurosaki T. (1998). J. Exp. Med., 187, 1355-1360.

Nadler MJS, Chen B, Anderson JS, Wortis HH and Neel BG. (1997). J. Biol. Chem., 272, $20038-20043$.

Nishizumi H, Horikawa K, Mlinaric-Rascan I and Yamamoto T. (1998). J. Exp. Med., 187, $1343-1348$.

O'Rourke L, Tooze R and Fearon DT. (1997). Curr. Opin. Immunol., 9, 324-329.

Ono M, Okada H, Bolland S, Yanagi S, Kurosaki T and Ravetch JV. (1997). Cell, 90, $293-301$.

Pleiman CM, D'Ambrosio D and Cambier JC. (1994) Immunol. Today, 15, 393-399.

Putney Jr JW and Bird GSJ. (1993). Cell, 75, 199-201.

Reth M and Wienands J. (1997). Annu. Rev. Immunol., 15, $453-479$.

Sanchez M, Misulovin Z, Burkhardt AL, Mahajan S, Costa T, Franke R, Bolen JB and Nussenzweig M. (1993). $J$. Exp. Med., 178, 1049-1055.

Saouaf SJ, Mahajan S, Rowley RB, Kut SA, Fargnoli J, Burkhardt AL, Tsukada S, Witte ON and Bolen JB. (1994). Proc. Natl. Acad. Sci. USA, 91, 9524-9528.

Scharenberg AM, El-Hillal O, Fruman DA, Beitz LO, Li Z, Lin S, Gout I, Cantley LC, Rawlings DJ and Kinet J-P. (1998). EMBO J., 17, 1961-1972.

Sugawara $\mathrm{H}$, Kurosaki $\mathrm{M}$, Takata $\mathrm{M}$ and Kurosaki $\mathrm{T}$. (1997). EMBO J., 16, 3078-3088

Takata M, Homma Y and Kurosaki T. (1995). J. Exp. Med., 182, $907-914$.

Takata M and Kurosaki T. (1996). J. Exp. Med., 184, $31-40$.

Takata M, Sabe H, Hata A, Inazu T, Homma Y, Nukada T, Yamamura $\mathrm{H}$ and Kurosaki T. (1994). EMBO J., 13, $1341-1349$.

Unkeless JC. (1979). J. Exp. Med., 150, 580-596.

Wang J, Koizumi T and Watanabe T. (1996). J. Exp. Med. 184, $831-838$

Yamanashi Y, Fukui Y, Wongsasant B, Kinoshita Y, Ichimori K, Toyoshima K and Yamamoto T. (1992). Proc. Natl. Acad. Sci. USA, 89, 1118-1122. 